\title{
Discapacidad, autonomía y reconocimiento intersubjetivo en el Sistema Nacional Integral de Cuidados
}

\author{
Sharon Carolina Díaz Fernández \\ Universidad de la República (UDELAR)
}

Discapacidad, autonomía y reconocimiento intersubjetivo en el Sistema Nacional Integral de Cuidados Resumen: En este artículo se estudia la generación de condiciones de autonomía y reconocimiento para personas en situación de dependencia mediada por Discapacidad, en el marco del Sistema Nacional Integrado de Cuidados (SNIC) implementado en Uruguay. Se analizan las conceptualizaciones que se plasman en los marcos discursivos institucionales, y en la vivencia cotidiana de los sujetos implicados, a los cuales se llega a través de la realización de entrevistas (en varios departamentos del país donde el Programa de Asistentes Personales se implementó como piloto). Se propone problematizar teóricamente cuáles son las implicancias entre formas de reconocimiento y potencialidad de autorreferencia individual (autoconfianza, autorespeto, autoestima) para personas en situación de dependencia mediada por Discapacidad; así como discutir si la forma de implementación del SNIC a través del componente Asistente Personal conlleva la posibilidad de ampliación de sus espacios de reconocimiento intersubjetivo y autonomía.

Palabras claves: Discapacidad. Autonomía. Reconocimiento. Cuidados.

Deficiência, autonomia e reconhecimento intersubjetivo no Sistema Nacional Integral de Cuidados Resumo: Este artigo analisa a criação de condições de autonomia e reconhecimento para pessoas em situação de dependência mediada por deficiência, no contexto do Sistema Nacional Integrado de Cuidados (SNIC) implementado no Uruguai. Analisa as conceituações configuradas nos contornos discursivos institucionais, e na vivência cotidiana dos sujeitos implicados, os quais são entrevistados (em várias localidades do país onde o Programa de Assistentes Pessoais foi implementado como um projeto piloto). Propõe-se problematizar teoricamente as implicações das formas de reconhecimento e potencialidade de autorreferência individual (autoconfiança, autorrespeito, autoestima) para pessoas em situação de dependência mediada por deficiência. Discute se a forma de implementação do SNIC através do componente Assistente Pessoal oferece oportunidades para a ampliação de seus espaços de reconhecimento intersubjetivo e autonomia.

Palavras-chave: Deficiência. Autonomia. Reconhecimento. Cuidados.

Disability, Autonomy and Intersubjective Recognition in the Integral National Care System

Abstract: This article analyzes the creation of conditions for autonomy and recognition of people in situations of dependency mediated by disability, within Uruguay's National Integral Care System (SNIC). It analyzes the conceptualizations that take form in the institutional discursive frameworks, and in the daily experience of the subjects involved, who are interviewed (in various regions of the country where the Personal Assistance Program is implemented in pilot form). It proposes to theoretically problematize the implications of forms of recognition and potentiality of individual self-reference (self-confidence, self-respect, self-esteem) for people in situations of dependency mediated by disability. It discusses if the form of implementation of the SNIC through the Personal Assistant component offers opportunities for expansion of their spaces of intersubjective recognition and autonomy.

Keywords: Disability. Autonomy. Recognition. Care. 


\section{Introducción}

En las últimas décadas, la temática de la discapacidad ha cobrado un especial emplazamiento y relieve a nivel global pero también en la particularidad de nuestro continente y en la especificidad del Uruguay. Uno de los engranajes que en el último tiempo se viene tensionando y redimensionando es la relación discapacidadcuidados. Prueba fehaciente de ello es la implementación reciente del Sistema Nacional Integrado de Cuidados (SNIC), y su presentación en tanto novel política pública que se delinea hacia tres específicas poblaciones: infancia, vejez y discapacidad. En esta coyuntura, el Grupo de Estudios de Discapacidad (GEDIS) ${ }^{1}$ se propuso la realización de un estudio exploratorio, de acercamiento a las voces y experiencias de los sujetos cuyas cotidianidades se vieron de alguna forma alcanzadas por la propuesta del SNIC: "Cuidados en el Uruguay: Subjetividades y objetividades en la implementación del Programa de Asistentes Personales."

Circunscripto en dicho marco, aquí se presenta el eje de análisis que vincula al SNIC en su Programa de Asistentes Personales, con los procesos de Autonomía y reconocimiento intersubjetivo en relación a personas en situación de discapacidad con dependencia. A estos efectos el documento se divide en tres secciones. En una primera parte se establecen los lineamientos metodológicos generales que guiaron la investigación más amplia. Una segunda sección propone analizar las conceptualizaciones de la discapacidad y Autonomía presentes en la propuesta del SNIC, retomando también algunos fragmentos de las entrevistas realizadas, para obtener un primer acercamiento a las percepciones que al respecto tienen las instituciones, familias, asistentes personales (AP) y personas en situación de dependencia mediada por discapacidad. La tercera parte propone problematizar teóricamente las potencialidades del SNIC en su Programa de AP para la ampliación de espacios de reconocimiento intersubjetivo y Autonomía para la persona en situación de dependencia mediada por discapacidad. Para el análisis se opta por retomar los ejes de la autorreferencia personal (autoconfianza, autorespeto, autoestima) en tanto iluminadores que habilitan a deconstruir la presencia (o no) de condiciones para la generación de un real reconocimiento intersubjetivo.

Culmina el documento con la presentación de algunas reflexiones finales que tiene por función abrir nuevos lineamientos para el debate y la búsqueda de otros rodeos analíticos posibles. Esto en el entendido de que se trata de una temática amplia y de sustancial relevancia al tocar - a través de esta política - aspectos que hacen a la cotidianidad actual, pero también a la capacidad de expandir el proyecto de sujetos que históricamente se han encontrado al margen de las condiciones para un ejercicio real de este derecho.

\section{Aspectos Metodológicos}

La propuesta de esta investigación es exploratoria y de corte cualitativa. La metodología utilizada para la deconstrucción analítica se asienta en la matriz histórico-crítica de pensamiento en base a la dialéctica hegeliana, y propone el estudio de lo real a través de la realización de sucesivos rodeos (KOSIK, 1969). Dichos rodeos se hacen necesarios para trascender lo más inmediato o fenoménico hacia la búsqueda de la esencia, y así ir delimitando el objeto de investigación al seleccionar ciertas determinaciones analíticas que habilitarán una entre muchas aproximaciones posibles a la totalidad inabarcable: "todo objeto percibido, observado o elaborado por el hombre es parte de un todo, y precisamente ese todo, no percibido explícitamente, es la luz que ilumina y revela el objeto singular, observado en su singularidad y en su significado.” (KOSIK, 1969, p. 43).

Para ello se retoma tanto lo plasmado en los marcos discursivos institucionales como las vivencias de la cotidianeidad de los sujetos directamente implicados en tanto elementos fenoménicos que se intentará trascender a través de la realización de rodeos teóricos de algunas determinaciones analíticas que se consideran relevantes. En cuanto al recabado de datos, la técnica preponderante fue la realización de entrevistas, abarcó varios departamentos del Uruguay (Salto, Paysandú, Artigas, San José, Florida, Rocha, Montevideo) e incluyó tanto a personas en situación de discapacidad (beneficiarios ${ }^{2}$ del programa), como a su familia y Asistente Personal (AP). A tales personas se llegó a través principalmente de la técnica de bola de nieve. ${ }^{3}$

El muestreo inicial, establecido en base al principio de factibilidad estaba pensado de la siguiente manera: 1 Primera Infancia (0 a 3) - Varón de Artigas; 1 Infancia (4 a 12) - Varón de Paysandú; 2 Adolescentes (13 a 18) - Mujer de Maldonado; Varón de Rocha; 2 Jóvenes (19 a 30) - Mujer de Canelones; Varón de Florida; 2 Adultos (31 a 64) - Mujer de Salto; Varón de San José; 2 Viejos (+ 65) - Mujer de Montevideo; Varón de Treinta y Tres. El muestreo realizado tuvo la mayor concordancia posible con el muestreo inicial, salvo los entrevistados adultos mayores y el cambio del niño de Artigas por dos niños de Paysandú con edades similares. Esto se debió a la ausencia de la población inicialmente seleccionada ante los datos recabados en los lugares concretos sobre beneficiarios/as del Programa de Asistentes Personales. A esto se sumó la utilización de fuentes documentales sobre el SNIC (URUGUAY, 2015a) y el Programa de Asistentes Personales (BANCO 
DE PREVISIÓN SOCIAL, 2017); y datos en torno a discapacidad del Instituto Nacional de Estadística (INE) - Censo 2011 (INE, 2011). Este estudio se integró por varios ejes temáticos que han dado lugar a un conjunto de profundizaciones teórico-reflexivas más amplias ${ }^{4}$, de las cuales el presente documento es parte. Para la publicación de este artículo en particular, se obtuvo aprobación tanto de la Coordinación del GEDIS como del resto de los investigadores, quienes han accedido a su lectura, previo a su postulación para evaluación.

Cabe destacar que la propuesta de investigación general así como este artículo en particular, se proyectó y concretó en el entendido de que es un tema relevante en nuestro contexto, a la vez que original y útil en la interrelación entre arena académica y arena política. Su desarrollo se orientó por los principios de integralidad en la investigación científica, dando relevancia a los aspectos éticos ${ }^{5}$ de trasparencia y fiabilidad, ya sea en el recabado como en la difusión de la información. En este sentido, todas las entrevistas fueron realizadas con previo acuerdo y consentimiento de las partes, toda vez que hubieron recibido por escrito, cuáles serían los objetivos y alcances que se proponían en la investigación. En caso de entrevistas realizadas a niños/as y adolescentes, las mismas se realizaron con previo consentimiento de sus referentes adultos, y en presencia de ellos (salvo solicitud de los propios entrevistados y aval de adulto responsable). Va de suyo que la pauta de preguntas ofició como una guía, con potencialidad de flexibilidad para adecuarse a los diferentes encuadres de entrevista (en función de las edades y situaciones de discapacidad), generando un proceso de investigación basado en el principio de respeto a la persona humana.

\section{Resultados y Discusión}

\section{a) Discapacidad y autonomía; discursos y prácticas mediados por ideología}

Actualmente coexisten en tensión diversos marcos desde donde se da forma y contenido a la discapacidad. Cada una de ellos implica fundamentos diversos desde lo teórico-metodológico, ético-político y epistemológico y se expresa en formas de nombrar, de comprender, deconstruir y responder con prácticas a esta temática. El GEDIS ha optado por un posicionamiento que adscribe a pensar la discapacidad en tanto constructo social, y por tanto toma distancia de la identificación de la discapacidad con el déficit, la responsabilización individual y encauzamiento de las diferencias. Conceptualizar la discapacidad en tanto producción socio-históricamente situada implica comprender su fuerte componente ideológico, es decir, llevar el análisis a una lectura en clave de ideología de la normalidad.

Siguiendo a Althusser, la Ideología ha de entenderse en tanto “'representación’ de la relación imaginaria de los individuos con sus condiciones reales de existencia." (ALTHUSSER, 1970, p. 43). Uno de los puntos interesantes de esta perspectiva, es el carácter material que adquiere la Ideología distanciándose de toda asociación entre ideología y mera idea: "la ideología tiene una existencia material. [...] en tanto esas ideas son actos materiales insertos en prácticas materiales, reguladas por rituales materiales definidos, a su vez, por el aparato ideológico material del que proceden las ideas de [...] [un] sujeto.” (ALTHUSSER, 1970, p. 47-50). Dentro de esta materialidad, se complejiza el sentido que la noción de Ideología adquiere, puesto que al ser también estructuras inconscientes y naturalizadas, las representaciones imaginarias de los individuos con sus condiciones reales de existencia son asimiladas y pensadas como producciones singulares, cuando en realidad son impuestos que operan hacia la reclusión de los individuos en sujetos. Ello resulta particularmente interesante en relación al engranaje discapacidad/cuidados puesto que en cuanto a la construcción de la noción discapacidad, la ideología se coloca en la intersección entre su producción histórico-social y su conceptualización como fenómeno natural, objetivo y evidentemente dado:

Este trabajo ideológico borra las huellas de los procesos históricos concretos de producción de la normalidad y en consecuencia de producción de la discapacidad como uno de los modos en que este sujeto, situación, cuerpo no se ajusta a la norma [...] El efecto de ideología que queremos desentrañar borra estos procesos y naturaliza el déficit por asignarle un carácter biológico, individual ahistórico. (ANGELINO, 2009, p. 135-136).

Trabajo ideológico que se opera en el marco de una forma de organización social, instituyente de pautas de relacionamiento tanto de las personas entre sí, como de estas y el contexto socio-histórico en el que se encuentran. Particularmente con el establecimiento del sistema capitalista adviene una forma de racionalidad en que la complejidad de lo real parece escindirse en dualidades: útil/inútil, incluido/excluido, sano/enfermo, normal/anormal. El producto fue la configuración de un proyecto de individuo homogéneo, unificado, normal; y también tuvo como contra-punto la producción específica de los individuos que hacían parte de la alteridad 
alejada de dichos estándares del deber ser. Resulta entonces interesante indagar en cuanto a la forma en que se comprende, vivencia y experiencia la discapacidad:

Sé que hoy en día tener Síndrome de Down no es tan oscuro como antes [...] pero estar todo el tiempo con algo que es diferente a uno y no tener las herramientas para brindarle todo lo que uno quiere es difícil. (Asistente Personal de niño de 1 año de edad, Paysandú, entrevista realizada en agosto 2015).

Entrevistador: ¿Volverías a trabajar con autismo si tuvieras la posibilidad? Entrevistado: Sí, hay gente que tiene miedo, que me ha dicho que ni loca trabaja en eso [...] mucha gente, gente conocida me decía, 'únicamente tu' [...] (Asistente Personal de adolescente varón de 13 años de edad, Rocha, entrevista realizada en junio 2015).

Estos fragmentos hacen a indicios de lo permeada que se encuentra la sociedad por formas de comprender y posicionarse en materia de discapacidad que remiten a la estandarización de lo correcto/normal para ser y estar, donde lo que se aparta de estos parámetros tiende a caracterizarse como oscuro, diferente, enfermo. No obstante esto, de las entrevistas realizadas también se desprende un giro a este posicionamiento, donde aflora la riqueza de colocar este enfoque normativo de la discapacidad en tensión con otras miradas:

en el jardín hemos logrado que no me vean como un extraño con otro extraño, soy uno más que estoy orientando y apoyando a un compañero y que cuando otro compañero precisa de algo también lo hago [...] Es un trabajo exigente, requiere de mucha creatividad y paciencia, creatividad porque la mayoría de las veces no aprenden de la forma que está estandarizada y tenés que encontrarle esa vueltita para que puedan aprender de esa otra forma, y paciencia porque estás en un sistema que no está preparado para la discapacidad, entonces tenés que enfrentarte con prejuicios, falta de voluntades, y ahí en esos casos actúo como de abogado [risas] voy defendiendo, legimitando no solo mi figura sino la presencia de M. en el jardín. (Asistente Personal de M., varón de 4 años de edad, Paysandú, entrevista realizada en agosto 2015).

Preferiría que esa plata la invierta en sensibilización sobre la temática, en jornadas de charlas, en campañas en los medios de comunicación, hay mucho prejuicio, estigmatización, y esa barrera cultural se derrumba con información [...] también se nota la falta de preocupación en la temática, es mi hijo, son personas, y el Estado con este tipo de cosas parece desmerecer esta problemática. Ah mira que problemática no quiere decir que mi hijo sea un problema para mí, el problema está en la sociedad en la que nació, que no conoce, que no sabe, y no lo juzgo, yo tampoco sabía nada, pero informándome aprendí, y aprendí mucho, crecí, y eso es lo que hace falta, información. (Madre de persona en situación de discapacidad, varón 4 años de edad, Paysandú, entrevista realizada en agosto 2015).

Tal vez esta lucha cultural que se esboza en los fragmentos seleccionados esclarece de lo que se trata; a la hora de comprender y conceptualizar la temática de la discapacidad desde un enfoque social y de derechos, se apunta a trascender una forma de racionalidad instrumental, dicotomizada y llevar la crítica también a la dimensión histórica e ideológica de su proceso. Los marcos normativos ${ }^{6}$ con que cuenta nuestro país se han acercado progresivamente a una conceptualización de la discapacidad con orientaciones tendientes (aunque se entiende que aún no suficientes) a este enfoque. Si se observa la definición dada por la Ley ${ }^{\circ} 18.651$ de Protección Integral a las Personas con Discapacidad se destaca:

Artículo $2^{\circ}$ - Se considera con discapacidad a toda persona que padezca o presente una alteración funcional permanente o prolongada, física (motriz, sensorial, orgánica, visceral) o mental (intelectual y/o psíquica) que en relación a su edad y medio social implique desventajas considerables para su integración familiar, social, educacional o laboral. (URUGUAY, 2010).

Más allá de que está presente la noción de persona en interacción con el medio social en que se encuentra y desenvuelve, persisten ideas vinculadas a lo deficitario. Es en esta interacción donde se instalan muchas veces las barreras más importantes para la integración que en el marco normativo se indica, dando lugar a la existencia de situaciones en que los apoyos requeridos son variados. En lo que respecta a este trabajo, el interés se centra en experiencias en que tal integración familiar, social, educacional o laboral se hace más compleja al tratarse de situaciones de dependencia mediadas por discapacidad. La ley arriba mencionada también normativiza al respecto, y establece la Asistencia Personal para Personas con Discapacidades Severas (Ley $\mathrm{n}^{\circ} 18.651$, cap. IV) (URUGUAY, 2010), incorporando ya en su artículo n ${ }^{\circ} 25$ la figura del Asistente Personal. En cumplimiento a lo 
dispuesto, en diciembre 2015 se publica la Ley n ${ }^{\circ} 19.353$ por la que se crea el Sistema Nacional Integrado de Cuidados, cuyo objetivo apunta a la promoción del desarrollo de la autonomía de las personas en situación de dependencia, su atención y asistencia. Incorpora como una de sus poblaciones ${ }^{7}$ objetivos a las personas con discapacidad que carecen de autonomía para desarrollar las actividades y atender por sí mismas sus necesidades básicas de la vida diaria (Ley $\mathrm{n}^{\circ}$ 19.353, artículo 8, inciso A, numeral 2) (URUGUAY, 2015b). Este marco normativo realiza una definición de cómo han de entenderse estos términos:

Autonomía: la capacidad de controlar, afrontar y tomar, por iniciativa propia, decisiones acerca de cómo vivir y desarrollar las actividades y necesidades básicas de la vida diaria, contemplando la cooperación equitativa con otras personas.

Dependencia: el estado en que se encuentran las personas que requieren de la atención de otras u otras personas o ayudas importantes para realizar actividades básicas y satisfacer las necesidades de la vida diaria. (Ley ${ }^{\circ}$ 19.353, artículo 3, inciso C y D). (URUGUAY, 2015b).

En el marco normativo que crea el SNIC autonomía e independencia aparecen en diversas partes, muchas veces colocados en una relación que al entender de quien suscribe, quita claridad al establecer la propuesta de AP en relación a personas que carecen de autonomía, cuando en realidad pueden ser personas autónomas según la definición que la propia ley plantea, pero absolutamente dependientes dada una situación de discapacidad severa. En la especificidad de lo que plantean los Decretos de reglamentación del Programa de AP, estos aspectos aparecen más despejados en una tendencia que apunta (y se enfatiza en el decreto 117/ 2016 como revisión al 214/2014) a establecer los AP para personas en situación de dependencia ${ }^{8}$ severa (URUGUAY, 2014, 2016). Estos aspectos se consideran sustanciales dado que ambos conceptos si bien pueden tener un uso similar en el vocabulario común, tienen implicancias diversas y deconstrucciones analíticas que recorren vías diferentes. En todo caso, se trataría de los pares dependencia/independencia y autonomía/ heteronomía. A los efectos de esta limitada exposición se centrará el análisis en la autonomía como determinación analítica con potencialidad de iluminar el estudio de los procesos de reconocimiento intersubjetivo para personas en situación de discapacidad que se analizará en el segundo momento de este trabajo.

Al estudiar la noción autonomía, una de las posibilidades es remitirse al ámbito de la Filosofía, donde se encuentra la misma se ha convertido en una de las referencias conceptuales más importantes en la filosofía moral contemporánea, cuya matriz proviene de Kant. Dicho autor sostiene entre otras cosas, que todo ser racional - gracias justamente a la razón - posee una "voluntad autónoma" capaz de "autolegislar en concordancia con la ley moral" de alguna forma trascendente a la idiosincrasia y a los intereses singulares del sujeto (PEREIRA, 2014, p. 12). En este sentido, el pensamiento ilustrado hace de la autonomía su consigna: "la condición necesaria y suficiente de un pensamiento que se autodetermina" (CASTILLO HERMOSO, 1999, p. 33). Ahora bien, con la Ilustración no se afirma esta noción aisladamente; por el contrario, el concepto de autonomía surge ligado a la idea de dignidad y de igualdad de los sujetos. Esta última deja de justificarse por cuestiones como el linaje, el honor, etc. y pasa a sentar sus bases en el entendido de que cada sujeto constituye un fin en sí mismo (PEREIRA, 2014).

La autonomía entonces también hace a una noción intersubjetiva: "consiste en el logro de una relativa capacidad de autodeterminación, de acción lúcida y apasionada, pero de una acción que es siempre interacción con otros (reales o virtuales)" (HELER apud MÍGUEZ, 2010, p. 185). Incluye por ende una doble dimensión, tanto lo que hace a la autodeterminación para afrontar la búsqueda de condiciones para realizar intereses personales como la "autonomía moral que remite al compromiso con la cooperación equitativa" (PEREIRA, 2014, p. 13). Y es allí que lo intersubjetivo se enfatiza y abre la posibilidad para avanzar sobre la perspectiva centrada en la autodeterminación y superarla en la incorporación del reconocimiento recíproco de base Hegeliana. Esta propuesta será principalmente desarrollada por Honneth quien frente a la moralidad kantiana se corre y coloca como punto de partida el conflicto:

Es así como se sirve de la ontología del poder de Foucault, traducida intersubjetivamente, para aportar una noción ética de conflicto que hunde sus raíces en la filosofía hegeliana [...] Hegel a la Moralität kantiana opone el concepto de Sittlichkeit, de eticidad: para él la autonomía no constituye un privilegio que se gana en oposición al mundo, sino una forma histórica que emerge de éste. (CASTILLO HERMOSO, 1999, p. 37).

En este sentido resulta provocativa la propuesta de Giusti (2007) al colocar autonomía y reconocimiento intersubjetivo entrelazadas en un mismo movimiento como momentos de una estructura compleja, en donde el momento del reconocimiento necesita y promueve la autonomía como instancia previa para que pueda producirse una relación igualitaria/conflictiva entre los sujetos; y la promueve dado que tiene sobre el individuo el efecto de 
fortalecer y enriquecer su identidad, es decir, su autonomía. "Mientras más exitosa sea la experiencia del reconocimiento, es decir, mientras más sólidos sean los lazos que unen al individuo con su comunidad, más posibilidades tiene él mismo de diferenciarse y de adquirir conciencia de su particularidad." (GIUSTI, 2007,p. 45). El proceso de alcanzar mayor autonomía entonces está ineludiblemente vinculado a las condiciones sociales en que los sujetos se inserten, las cuales han de propiciar o desalentar su generación. En párrafos anteriores se colocó que en el marco del SNIC el objetivo radica en la promoción de autonomía de las personas en situación de dependencia. De acuerdo al desarrollo analítico realizado, tal objetivo es ambiciosamente necesario al colocarse en relación a personas en situación de dependencia mediada por discapacidad. Necesidad que se vivencia y expresa desde las voces de quienes cotidianamente encuentran condiciones contextuales no disponibles para la generación de esa estructura compleja entre autonomía y reconocimiento:

Entrevistador: ¿Hace mucho que estás sin la silla de ruedas eléctrica? Entrevistado: Y hace un par de semanas; lo que pasa que un par de semanas así digo, es como si pasara un tiempo bastante porque uno tiene que siempre depender de alguien [...] Pero trabajar no puedo porque no tengo la silla no puedo, una traba importante [...] Porque la ayuda para uno que está solo en la casa... ya te digo en principio siempre precisé, digo, tenías que estar pagando, tenías que estar sacando dinero o que alguien te ayudara a pagar para que te dieran ayuda [...] yo pagaba una persona y a veces no me daba ni para comer. (Hombre en situación de discapacidad, 39 años de edad, San José, entrevista realizada en agosto 2015).

Ante la desesperación, uno intenta, prueba, con tal de hacer todo lo que esté a nuestro alcance para que pueda ir a la escuela, pueda recibir educación y logre autonomía cuando sea grande, que ese es mi objetivo. (Madre de persona en situación de discapacidad, varón 4 años de edad, Paysandú, entrevista realizada en agosto 2015).

Yo lo vivo en carne propia, yo hace pila que tenía esa ilusión de que capaz que puede llegar algo, para lo que queremos cierta libertad [...] Como que quería cierta libertad yo también, es como que buscar lo necesario y también poder buscar cierta independencia, dentro de la dependencia. (Mujer en situación de discapacidad, 33 años de edad, Salto, entrevista realizada en junio 2015).

No se trata de una negación a la situación de dependencia, que es claramente reconocida por las personas entrevistadas. La diferencia entre los conceptos de dependencia y autonomía, queda plasmada no solo en el análisis teórico sino también en las voces que expresan y reclaman la necesidad de que en el marco de políticas estatales se den respuestas para la asistencia y atención de sus necesidades cotidianas, como parte de sus derechos:

Entrevistador: ¿Cuáles fueron los motivos que impulsaron a realizar las gestiones para solicitar el servicio? Entrevistado: $\mathrm{Y}$ en realidad yo antes, no estaba tan dependiente como estoy ahora antes me bañaba sola me vestía sola dentro de lo que podía, entonces de un tiempo a acá como que necesite más dependencia, para la higiene personal para todo en realidad. (Mujer en situación de discapacidad, 33 años de edad, Salto, entrevista realizada en junio 2015).

Entrevistador: ¿En qué cosas de la cotidianidad ves cambios? Entrevistado: Cosas diarias... capaz que a veces son muy comunes para otras personas pero para mí por ejemplo yo llego y tengo la casa limpia, tengo todo [...] y eso ya de por sí es un avance terrible, digo a uno, si me quiero bañar hoy me baño hoy [...] le digo bueno, vamos a bañar y ta [...] Entrevistador: ¿Antes cómo te organizabas con eso? Entrevistado: Y antes a veces, cuando la persona tenía ganas, claro es muy [...] es difícil, complicado. (Hombre en situación de discapacidad, 39 años de edad, San José, entrevista realizada en agosto 2015).

Tal como se plantea en otros documentos, se entiende que la posibilidad de reflexionar acerca de la autonomía permite realizar otros rodeos analíticos y también "objetivarse en la concepción de libertad, de autodeterminación, de reconocimiento" (MÍGUEZ et al., 2016, p. 75). En este sentido, no se trata de conceptos que tengan injerencia solamente en lo singular, sino que hacen a procesos que son siempre y de todas formas relacionales e intersubjetivos, y en los que por ende se interpela a todo el colectivo social sin excepción.

\section{b) Procesos de reconocimiento intersubjetivo; iluminador para el análisis del SNIC.}

En el desarrollo hasta aquí realizado se planteó un rodeo posible para la deconstrucción analítica de las nociones de discapacidad y autonomía, a los efectos de estudiar los procesos que se vienen generando en el 
marco de implementación del SNIC en la particularidad de su Programa de AP. Resta ahora centrar la atención en la problematización de las potencialidades del SNIC en su Programa de AP para la ampliación de espacios de reconocimiento intersubjetivo para la persona en situación de dependencia mediada por discapacidad. Dentro de este análisis se opta por dar centralidad a los ejes de autorreferencia individual (autoconfianza, autorespeto y autoestima) en el entendido de que ofician como llaves con capacidad de nexo entre los procesos de Reconocimiento intersubjetivo y la generación de mayor autonomía; "autoconfianza, autorespeto y autoestima son las llaves que abren y despliegan nuestra autonomía. Si dichas capacidades están socialmente situadas en cada esfera de reconocimiento, ser reconocido es empíricamente necesario para ser autónomo." (FASCIOLI, 2008, p. 24). Para ello se toma como eje central la propuesta de Honneth (1997) quien claramente da cuenta de sus bases: Hegel y G. H. Mead; lo que ambos autores plantean de forma independiente y que Honneth retoma en su propuesta es la distinción de tres formas de reconocimiento recíproco correspondientes a pares de interacción, a los que a la vez corresponde un potencial diverso de desarrollo moral y autorreferencia individual:

[...] de la dedicación emocional, que conocemos en las relaciones de amor o de amistad, se distinguen el reconocimiento jurídico y la adhesión solidaria, en tanto que formas de reconocimiento específicas. [...] con cada estadio de respeto recíproco, crece la autonomía subjetiva del singular. Pero sólo en Mead se otorga a la intuición así definida la articulación sistemática de una hipótesis empírica, conforme a la que, en la secuencia de las tres formas de reconocimiento, crece progresivamente el grado de relación positiva de la persona consigo misma. (HONNETH, 1997, p. 116).

La diferenciación en estas tres esferas de Reconocimiento intersubjetivo (amor, derecho y solidaridad) es de sustancial relevancia en la propuesta de Honneth. De su materialización devienen las tres autorreferencias personales arriba planteadas, que no son otra cosa que modos de integralidad personal que en suma

conforman la autorrealización del individuo como persona que se sabe autónoma, individualizada y susceptible de identificarse con ciertos objetivos más amplios. Concepto de persona intersubjetivo en tanto sí mismo que se encuentra en el otro mediante relaciones de reconocimiento no distorsionadas. (CASTILLO HERMOSO, 1999, p. 37).

Ahora bien, cuando lo que se materializa son relaciones de reconocimiento distorsionadas lo que se produce son tres formas correspondientes de no-reconocimiento o formas de menosprecio cuya identificación resulta sustancial como insumo para la problematización de diferentes temáticas, incluyendo el análisis de la discapacidad.

La referencia al reconocimiento intersubjetivo en las relaciones interpersonales primarias queda colocada bajo el tipo de reconocimiento del amor. Allí se incluyen relaciones de pareja, amistades, relaciones padreshijos y familiares, en donde la característica principal es la existencia de fuertes lazos afectivos entre los sujetos. Este primer estadio de reconocimiento recíproco resulta de vital importancia, dado que tiene por principio la capacidad de equilibrio entre simbiosis y autoafirmación, y en su culminación lleva a que los sujetos recíprocamente se confirmen como necesitados uno del otro y se reconozcan corporalmente en esa necesidad mutua. La capacidad de ser solo que retoma Honneth (1997) como indicio de autoconfianza se encuentra en retroalimentación con la permanencia de un deseo de fundirse con otra persona que es reconocida como persona autónoma. De esta forma, la forma de reconocimiento amor, que Hegel había descrito como "un 'sersí-mismo en otro', designa no una situación intersubjetiva, sino un arco de tensiones comunicativas que continuamente mediatiza la experiencia de poder-ser-solo con la de la fusión [...] que conexionados posibilitan un recíproco estar-junto-a-sí en el otro" (HONNETH, 1997, p. 130). Como condición favorable ante procesos de real reconocimiento intersubjetivo en las relaciones primarias, resulta que los sujetos involucrados generan en su construcción identitaria elementos de autoconfianza. Ahora bien, ¿qué sucede cuando tales procesos de reconocimiento no se dan, o se producen de forma falsa? Estaríamos ante una forma de menosprecio que también concierne a la corporalidad pero en clave ya no de satisfacción afectiva sino de sufrimiento; el no reconocimiento en las relaciones afectivas estaría implicando la amenaza contra la integralidad corporal, el retiro violento de la posibilidad del sujeto de disponer de sí mismo representando el modo elemental de humillación personal, de vergüenza y de perdida de la autonconfianza individual (HONNETH, 1997).

Volviendo entonces al SNIC en su Programa de AP, ¿se están dando condiciones tendientes a la generación de autoconfianza para personas en situación de dependencia mediada por discapacidad? A grandes rasgos, de los discursos de los AP se desprende una importante cuota valorativa y de afecto en relación a la persona con la que desenvuelven sus tareas. Muchas veces asumen esta función por vínculos previos de confianza desarrollados con la familia o la persona misma, o por inclinación personal al contar con familiares en situación de discapacidad. 
El segundo ámbito de reconocimiento que aborda Honneth (1997) es el del derecho. En este sentido, se parte de la perspectiva normativa de otro que ya no es un referente individualizado con el que se mantiene una relación afectiva, sino un otro generalizado mediante el cual se arriba al reconocimiento de uno mismo como persona de derecho, contando con la seguridad de realización de ciertas de sus pretensiones. Partiendo del amor como espacio para la afirmación de la autoconfianza, y estando ligado al presupuesto individual de la atracción por el otro, ante la ausencia de este estado la persona singular quedaría librada a voluntades individuales del reconocimiento o no del otro en su diferencia. El derecho aparece entonces como demarcación para la estructuración del reconocimiento más allá de las (in)voluntades individuales. El derecho se introduce como una forma de reconocimiento recíproco, bajo el supuesto y el convencimiento que todos los seres son iguales y libres, que obedecen a la misma ley en una decisión racional acerca de normas morales en función de su autonomía individual. En situación de presencia de reconocimiento en el derecho, lo que se genera es una idea de pertenencia del ciudadano en condiciones de igualdad, en tanto miembro pleno de una comunidad política con independencia de la posición o poder económico. Siguiendo a Mead, lo que se produce es una elevación de la facultad de referirse a sí mismo como persona moralmente responsable, con capacidad de elaborar juicios autónomos al estar cubiertos por derechos individuales que lo reconocen en tanto persona libre, y por ende una condición intersubjetiva de autorespeto. ¿Qué sucede ante la ausencia o falso reconocimiento en relación a las relaciones de derecho? "Vivir sin derechos individuales significa, para el miembro de la sociedad, no tener ninguna oportunidad para la formación de su propia autoestima." (HONNETH, 1997, p. 147). En tal sentido, se pasaría a una relación de desposesión del estatus de ser sujeto de interacción moralmente igual y plenamente valioso. Para la persona en su singularidad, la privación de sus pretensiones de derecho socialmente válidas, implica ser lesionado en sus expectativas de ser reconocido en tanto capaz de formación de juicios morales válidos, llevando a una forma de menosprecio que culmina con la pérdida de respeto de la persona sobre sí misma.

Como se expresó en párrafos anteriores, a nivel del derecho se han establecido diversos marcos normativos en materia de discapacidad. Sin duda se ha dado un progresivo acercamiento a una forma de comprender y posicionarse frente a la temática tendiente a generar condiciones más favorables a este segundo momento de reconocimiento intersubjetivo. No obstante esto, quedan aún cuestiones que deberán ser reconsideradas y tal vez transformadas para que se habiliten condiciones reales a la generación de una autorreferencia personal de autorespeto en las personas en situación de dependencia mediada por discapacidad. Las formas de nombrar y las condiciones para un real acceso a la información son algunos de los escollos que se deberán sortear para evitar generar un falso reconocimiento.

La última esfera de reconocimiento corresponde a la necesidad de ser valorado socialmente con relación a cualidades y facultades concretas, más allá de si están contempladas en el amor (como dimensión más subjetiva y fluctuante) o en el derecho (como reconocimiento jurídico, que también involucra elementos meramente discursivos). A esta relación recíproca de reconocimiento dada por la solidaridad, Hegel la denomina eticidad; allí se ponen en relieve las capacidades vitalmente desarrolladas por la persona, abriéndose modos diferenciados de autorrealización personal en el marco de un determinado escenario sociohistórico y cultural que colocará el horizonte de valoración. En este sentido, el valor social determinado con que será reconocido o no cada persona concreta es medido a partir del grado por el que se contribuye a la realización de los

Se hace necesario que como

colectivo social se apunte sostenidamente a la visibilización y comprensión de las personas en situación de discapacidad como sujetos de derechos, de potencialidades $y$ capacidades. Y de la mano de ello se coloca la necesidad de generar políticas y programas que apunten como el SNIC, a la promoción de la autonomía de cada uno/a, en un marco de reconocimiento recíproco real. objetivos sociales, devolviendo una referencia positiva hacia la propia persona en una autorrelación que podría caracterizarse como autoestima; "las relaciones de las valoraciones sociales, en las sociedades modernas, están sometidas a una lucha permanente, en la que los diferentes grupos, con los medios simbólicos de la fuerza, intentan alzar a objetivos generales el valor de las capacidades ligadas a su modo de vida." (HONNETH, 1997, p. 155). ¿Qué sucede ante la falta de solidaridad así entendida? Se producen sentimientos contrarios a 
los de honra y dignidad; la forma de menosprecio aquí correspondiente implica deshonra, injuria, el no poder atribuir al modo de vida que ostenta un sentido de valoración genérico.

Tal vez este momento de la propuesta de reconocimiento intersubjetivo sea la que genere más interpelaciones. ¿Se considera que las personas en situación de dependencia mediada por discapacidad pueden aportar de alguna forma a los objetivos que socialmente se estiman valiosos? ¿Cuáles son estos objetivos y quiénes son los sujetos que aportan a su logro? Más allá de la situación de dependencia, ¿socialmente se entiende que son personas con capacidades y cualidades significativas? Si se parte de la base de una sociedad regida por la lógica del sistema capitalista, muchas veces los objetivos colectivamente estimados quedan asociados a la utilidad, la eficiencia, el éxito laboral, la capacidad de consumo. No hace falta entonces profundizar en el análisis, para encontrar que las personas en situación de dependencia mediada por discapacidad no encuentran condiciones plenas para la generación de autoestima como autorreferencia del momento de reconocimiento que hace a la solidaridad. Sin embargo, la implementación de políticas y programas como el SNIC en la especificidad del Programa de AP aporta en la construcción de puentes alternativos, de matices e intersticios con los que habilitar la búsqueda de quiebres para la transformación. Se deja solo una cita, ilustrativa de esto que se enuncia, pero que adquiere mayor peso al representar la voz concreta de quién vivencia cotidianamente estos procesos:

el estar incluido y el sentirte que podes y el sentir que sos uno más que no hay diferencia de nada como yo decía una tenemos apoyo con sicóloga yo nunca me encontré como que era yo o el grupo no, yo y el grupo era una cosa sola. (Mujer en situación de discapacidad, 33 años de edad, Salto, entrevista realizada en junio 2015).

\section{Reflexiones Finales}

Poner bajo consideración y análisis teórico-reflexivo el engranaje discapacidad-cuidados se presenta como pertinente y necesario dado nuestro contexto y sus condiciones político-económicas y socio-culturales. Sin duda se trata de una propuesta ambiciosa, abarcadora y compleja pero de sustancial relevancia para los sujetos que la encarnan cotidianamente. El presente trabajo hace a una parte del estudio que se viene realizando desde el GEDIS en esta materia. Otras cuestiones esenciales para la deconstrucción y comprensión del SNIC, tales como su esencia y características en tanto política pública, la figura y el rol del AP, el análisis del concepto mismo de cuidados así como su feminización, son abordados en documentos paralelos que en armonía articulan la propuesta completa. En este caso, el eje se coloca en la interpelación al SNIC en su programa de AP para la generación de procesos tendientes al logro de mayor autonomía y reconocimiento intersubjetivo para las personas en situación de dependencia mediada por discapacidad. De lo hasta aquí avanzado se destaca un proceso positivamente superador, en el marco de condiciones de mayor apertura y crítica ante la temática de la discapacidad. Esto se considera sustancial dado que implica de alguna forma comenzar a romper con los supuestos de naturalidad y ahistoricidad que a través de la ideología de la normalidad se imponen. Sin dar pasos estos primeros pasos, el resto se vuelve más complejo. Se hace necesario que como colectivo social se apunte sostenidamente a la visibilización y comprensión de las personas en situación de discapacidad como sujetos de derechos, de potencialidades y capacidades. Y de la mano de ello se coloca la necesidad de generar políticas y programas que apunten como el SNIC, a la promoción de la autonomía de cada uno/a, en un marco de reconocimiento recíproco real.

De las entrevistas y los relatos obtenidos a lo largo del trabajo de campo realizado en 2015, se destaca a grandes rasgos una visualización positiva tanto de los AP, las familias y las personas en situación de discapacidad. Quizá como elemento más reiterado aparece el vínculo afectivo que prima en las relaciones establecidas, coadyuvando a la generación de condiciones para la afirmación de la autoconfianza. El reconocimiento intersubjetivo en el momento del derecho y de la solidaridad se viene reforzando, aunque como ya se expresó, restan transformaciones importantes tanto desde los marcos normativos y sus conceptos como desde la sociedad misma. Especialmente por esto último es que se entiende fecundo introducir el concepto de reconocimiento, pues desde allí se realiza un aporte para decodificar las pugnas y luchas sociales que incluyen la doble dimensión de la autoconcepción de uno mismo y la concepción del otro como sujetos dignos de derechos y deberes. En este sentido, cuando se visualiza lucha de los colectivos por sus derechos, cuando se percibe presión e interés en una temática en la agenda pública, puede estar sintomáticamente expresando la existencia de un falso reconocimiento, o una forma inadecuada del mismo que motiva subjetivamente a cuestionar y buscar las transformaciones que sean necesarias. 


\section{Referencias}

ALTHUSSER, L. Ideología y aparatos ideológicos del Estado. Freud y Lacan. Buenos Aires: Nueva Visión, 1970.

ANGELINO, M. A. Ideología e ideologia de la normalidade. In: ROSATO, A.; ANGELINO, M. A. (Coord.). Discapacidad e ideología de la normalidad. Desnaturalizar el déficit. Buenos Aires: Noveduc, 2009. p. 133-154.

BANCO DE PREVISIÓN SOCIAL. Programa de Asistentes Personales. 10 jan. 2017. Disponible en: <https://www.bps.gub.uy/ 9973/programa-de-asistentes-personales.html>. Acceso en: 10 nov. 2016.

CASTILLO HERMOSO, J. M. De la autonomía a la identidad: la lucha por el reconocimiento. THÉMATA: Concepciones y narrativas del yo, n. 22, p. 33-39, 1999. Disponible en: <http://institucional.us.es/revistas/themata/22/03\%20castillo.pdf $>$. Acceso en: 10 nov. 2016.

FASCIOLI, A. Autonomía y reconocimiento en Axel Honneth: un rescate de El Sistema de la Eticidad de Hegel en la filosofía contemporánea. Revista ACTIO, Montevideo, n. 10, p. 21-25, dic. 2008. Disponible en: $<$ http://www.actio.fhuce.edu.uy/Textos/10/Fascioli10.pdf $>$. Acceso en: 10 nov. 2016.

GIUSTI, M. Autonomía y reconocimiento. Ideas y Valores, Bogotá (Colombia), n. 133, p. 39-56, abr. 2007. Disponible en: <http:// www.scielo.org.co/pdf/idval/v56n133/v56n133a03.pdf>. Acceso en: 10 nov. 2016.

HONNETH, A. La lucha por el reconocimiento. Por una gramática moral de los conflictos sociales. Barcelona: Crítica, 1997.

INSTITUTO NACIONAL DE ESTADÍSTICA. Censo 2011. Montevideo, Uruguay, 2011. Disponible en: <http://www.ine.gub.uy/ web/guest/discapacidad $>$. Acceso en: 10 nov. 2016.

KOSIK, K. Dialéctica de lo concreto. México: Ed. Grijalbo, 1969.

MÍGUEZ, M. N. Libertad y autonomía ¿versus? disciplinamiento. Fronteras, Montevideo, v. 6, p. 38-44, 2010.

et al. Cuidados en el Uruguay. Entre subjetividades y objetividades. A un año de la implementación del Programa de Asistentes Personales. Revista Regional de Trabajo Social, Montevideo, n. 66, p. 62-79, 2016. Disponible en: <http://www.revistatrabajosocial.com/ >. Acceso en: 10 nov. 2016.

PEREIRA, G. El espacio normativo de la autonomía como realización de la igualdad. Santiago de Chile: CEPAL, 2014. (Serie Políticas Sociales, n. 191). Disponible en: <http://repositorio.cepal.org/bitstream/handle/11362/36628/1/S2014116_es.pdf>. Acceso en: 10 nov. 2016. URUGUAY. Decreto Presidencial n 117, de 25 de abril de 2016. Reglamentación de la Ley 19.353 relativo a la creación del Sistema Nacional Integrado de Cuidados (SNIC). Servicio de Asistentes Personales para cuidados de larga duración para personas en situación de dependencia severa. Centro de Información Oficial, Montevideo, 4 mayo 2016. Disponible en: <https://www.impo.com.uy/bases/ decretos/117-2016>. Acceso en: 10 nov. 2016.

Decreto Presidencial n ${ }^{\circ} 214$, de 28 de julho de 2014. Reglamentación del art. 25 de la Ley 18651 relativo a la protección integral de personas con discapacidad. Centro de Información Oficial, Montevideo, 1 ago. 2014a. Disponible en: <https://www.impo.com.uy/ bases/decretos/214-2014>. Acceso en: 10 nov. 2016.

. Ley n ${ }^{\circ} 18.418$, de 20 de noviembre de 2008. Convención sobre los Derechos de las Personas con Discapacidad. Diario Oficial de La República Oriental del Uruguay, Poder Legislativo, Montevideo, 4 dic. 2008. Disponible en: $<$ https://legislativo.parlamento.gub.uy/ temporales/leytemp3223895.htm>. Acceso en: 10 nov. 2016.

. Ley n ${ }^{\circ} 18.651$, de 19 de febrero de 2010. Protección Integral de los Derechos de las Personas con Discapacidad. Diario Oficial de La República Oriental del Uruguay, Poder Legislativo, Montevideo, 9 mar. 2010. Disponible en: <http://www.uncu.org.uy/downloads/ documents/201301240305059425.pdf>. Acceso en: 10 nov. 2016.

. Ley n ${ }^{\circ}$ 18.776, de 15 de julio de 2011. Adhesión de la República al Protocolo Facultativo de la Convención sobre los Derechos de las Personas con Discapacidad. Diario Oficial de La República Oriental del Uruguay, Poder Legislativo, Montevideo, 12 ago. 2011. Disponible en: <https://legislativo.parlamento.gub.uy/temporales/leytemp5096446.htm>. Acceso en: 10 nov. 2016.

Ley $n^{\circ} 19.353$, de 18 de noviembre de 2015. Sistema Nacional Integrado de Cuidados. Diario Oficial de La República Oriental del Uruguay, Poder Legislativo, Montevideo, 27 nov. 2015b. Disponible en: <http://www.rcj.com.uy/CMS/plugins/tinymce/plugins/ jfilebrowser/archivos/20151209081436_0.pdf>. Acceso en: 10 nov. 2016.

Ministerio de Desarrollo Social. Cuidados como Sistema. Propuesta para un modelo solidario y corresponsable de Cuidados en Uruguay. Montevideo: MIDES; DNPS, 2014b. Disponible en: <http://www.sistemadecuidados.gub.uy/innovaportal/file/34676/1/ cuidados_como_sistema.pdf $>$. Acceso en: 10 nov. 2016.

Junta Nacional de Cuidados. Plan Nacional de Cuidados 2016-2020. Montevideo: Junta Nacional de Cuidados, dic. 2015a. Disponible en: <http://www.sistemadecuidados.gub.uy/innovaportal/file/61181/1/plan-nacional-de-cuidados-2016-2020.pdf $>$. Acceso en: 10 nov. 2016.

VALLES, M. S. Técnicas cualitativas de investigación social. Reflexión metodológica y práctica profesional. Madrid: Editorial Síntesis, 2000.

\section{Notas}

1 Actualmente el GEDIS se conforma por integrantes de la Facultad de Ciencias Sociales (FCS), el Centro Universitario Regional Este (CURE) y el Centro Universitario del Litoral Norte (RN), todos espacios de la UDELAR que al encontrarse en marcos territoriales diversos acrecientan la riqueza de miradas y aportes sobre las realidades diferenciales dentro del país. 
2 Los documentos oficiales usan esta forma de nombrar; en la reglamentación del Programa de Asistentes Personales. El Decreto n 214/2014, art. 4 establece: "Será beneficiario del Programa [...]”"(URUGUAY, 2014a). La forma de nombrar cambia en el Decreto 117/2016 derogando el anterior y colocando en el artículo 5 la idea de usuario. Desde el GEDIS respetamos esta forma de nombrar institucionalizada más allá que por nuestro posicionamiento teórico-metodológico y ético-político desde la razón dialéctica adscribimos a la forma de persona o de sujeto (URUGUAY, 2016).

3 Se toma como criterio de selección para la muestra de personas a entrevistar, la técnica de la bola de nieve (VALLES, 2000), dónde los diferentes contactos se consiguen por mediación de los propios entrevistados. Mediante este criterio de selección metodológica, se intentó abrir el abanico de entrevistados, incluyendo personas de diferente sexo, edad, etc.

4 Para una lectura integral de la propuesta se sugiere el artículo de Míguez et al. (2016) que se señala en la bibliografía.

5 Es de señalar que la investigación general desarrollada por el GEDIS, de la cual surge el eje de profundización analítica que aquí se presenta, no requirió presentación anteComité de Ética. A su vez, de lo indagada y del conocimiento que se tiene a nivel de disposiciones y normas, no se cuenta en el país con legislación que impida o arbitre sobre la posibilidad de realizar entrevistas a personas en situación de discapacidad, al menos para aquellos casos en que no media declaración de incapacidad, por lo que el sujeto goza de sus derechos plenos en tanto que persona.

6 Cabe recordar que en Uruguay fue ratificada en el año 2008 la Convención de los Derechos de las Personas con Discapacidad (CDPD) de Naciones Unidas (NU), del año 2006, en el marco de la Ley no 18.418 y su Protocolo Facultativo en el año 2011 (Ley nº 18.776) (URUGUAY, 2008, 2011).

7 También se establecen como beneficiario a "niños y niñas hasta 12 años y personas mayores de setenta y cinco años que carecen de autonomía para desarrollar las actividades y atender por sí mismas sus necesidades básicas de la vida diaria“ (Ley n 19.353, artículo 8, inciso A, numeral 1 y 3) (URUGUAY, 2015b).

8 Desde los marcos institucionales se define la situación de dependencia en discapacidad como: "Ya sea que se trate de discapacidades físicas, intelectuales, mentales o múltiples, con la condición de que necesiten de otra persona en forma permanente, para poder desarrollar los actos corrientes de la vida diaria." (URUGUAY, 2014b, p. 2).

\section{Sharon Carolina Díaz Fernández}

sharon.diazfer@gmail.com

Licenciada en Trabajo Social por la Facultad de Ciencias Sociales de la Universidad de la República (UDELAR) Integrante del Grupo de Estudios sobre Discapacidad (GEDIS) de la Facultad de Ciencias Sociales de la Universidad de la República (UDELAR)

\section{GEDIS}

gedis@cienciassociales.edu.uy

Constituyente 1502 esquina Martínez Trueba

Montevideo - Uruguay 